\title{
Data types and different ways to store data in $\mathbf{R}$
}

Fu-Sheng Chou, MD, PhD

Data, Information, and Knowledge:

In the realm of information sciences, there is a well-established information pyramid as illustrated in Figure 1. (1) Data sits at the bottom, is in a large quantity; interpretation of data brings information that the human mind can process and use to form knowledge. Here is a brief example:

Data are quantified observations that by themselves show no meaning -

"10" in the statement of ten apneas in the past $24 \mathrm{hrs}$,

Information is the interpretation of a multitude of data -

Ten apneas in the past 24 hrs with feeding intolerance, and

Knowledge is a plausible interpretation of information gathered based on established facts or knowledge -

New-onset frequent apnea episodes with feeding intolerance may be a sign of systemic infection.

\section{"In medical school, we learned about medical knowledge. In residency, we learned to apply the medical knowledge to practical patient care, and in fellowship, through scholarly activity, we started to get deep into data and information."}

In medical school, we learned about medical knowledge. In residency, we learned to apply the medical knowledge to practical patient care, and in fellowship, through scholarly activity, we started to get deep into data and information. Modern medicine is built upon this information pyramid (Figure). Now, with artificial intelligence, the circles are going to roll even faster.

Data science is a field that deals with data, which is the element in the pyramid with which we have the least contact. A data scientist would say, $80 \%$ of data science work is cleaning up the data. This is how terrible we, as amateurs, are when we hand over a table to our analytics colleagues and friends.

Hadley Wickham, the author of $R$ for Data Science and many books, the creator of the tidyverse package and many others, and the chief scientist at RStudio, introduced the concept of tidy data, which he defined as "a consistent way to organize your data in $R$," in the introduction of Chapter 12 in $R$ for Data Science. (2) A famous line by Dr. Wickham, in a reflection to a famous quote by the Russian writer Leo Tolstoy, states:

Tidy datasets are all alike, but every messy dataset is messy in its own way.

There are various types of data (e.g., number, character, datatime, etc.), and each programming language deals with data types slightly differently. Luckily, to me at least, it is quite intuitive when it comes to data type recognition and management in R. Getting the data type right is the first step towards successful projects and a bright future with $\mathrm{R}$.

\section{"Getting the data type right is the first step towards successful projects and a bright future with $R^{. "}$}

Tidy data is not just about data type; instead, its spirit lies in the word "organize." Organizing data requires grouping them into containers (the invisible ones). A data table is a container; a vector is also a container. There are various types of containers built-in R. Choosing the right one to store your data is important.

So, I think, before we start building our first webApp for the neonatal abstinence score, we should first introduce data types and containers. Let's get started!

Working with data types in $\mathrm{R}$

Basic data types:

- Numeric: This is the data type for continuous variables, for example, urine output.

- Integer: Integer can be thought of as a subtype of numeric, but more importantly, use it on variables that do not allow fractions, e.g., neonatal abstinence score, length of hospital stay, N-PASS scores.

- Logical: The Yes' and the Noes. This is the data type for comparisons.

- Character: This is the data type for text.

- Factor: Factors are a special data type that is useful for categorical variables. The beauty of the factor data type is the ability to re-level the variables. Please see below.

\section{Data type conversion:}

In $\mathrm{R}$, you can convert data types by using a group of functions that start with "as.": as.xxxxx(). For example:

$>\quad \mathrm{a}=1.2$

$>$ as.numeric(a)

[1] 1.2 


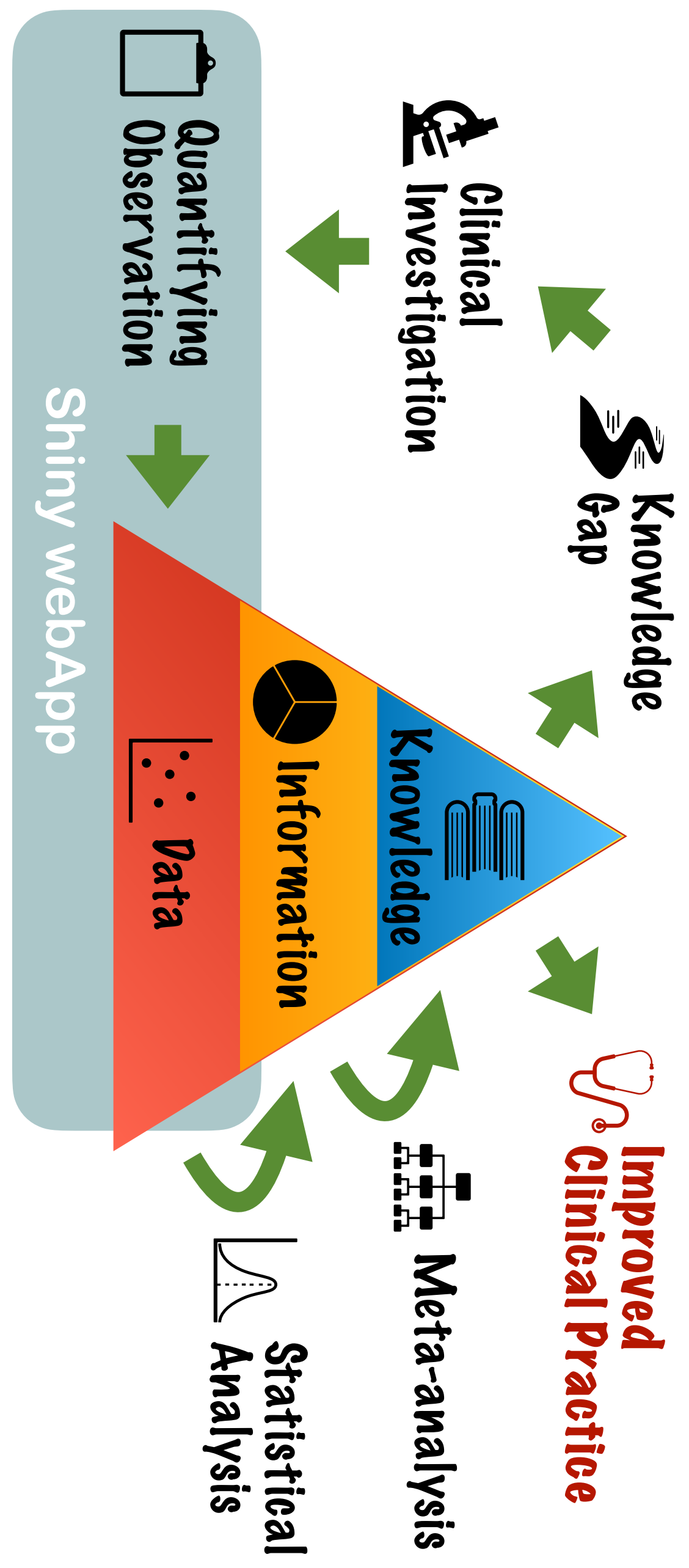


$>$ as.character(a)

[1] "1.2"

$>\quad$ as.factor(a)

[1] 1.2

Levels: 1.2

$>\quad$ as.integer(a)

[1] 1

To check the data type, use a group of functions that start with

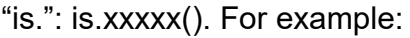

$>\quad a=$ as.factor(1.2)

$>a$

[1] 1.2

Levels: 1.2

$>$ is.factor(a)

[1] TRUE

$>$ is.numeric(a)

[1] FALSE

Now, try and type as.numeric(a), and see what happens:

$>$ as.numeric(a)

[1] 1

Why do we get the result of numeric 1 back? It is because 1.2 is the first level of the factor (well, there is only 1 level), so, when you convert a factor into a numeric data type, R converts the "level" of the factor, not the "content" of the factor. See more examples below when I show you multi-level factors stored in a container.

"Try and convert data types back and forth. Come up with simulated data content that your research projects will have. Convert data types back and forth. If you run into errors, Google the error messages. Alternatively, you can email me."

Try and convert data types back and forth. Come up with simulated data content that your research projects will have. Convert data types back and forth. If you run into errors, Google the error messages. Alternatively, you can email me.

Containers for data storage

In the above example, when we typed $>a=1$ earlier, $a$ is the container or an object, and 1 is the content that was placed in the container $a$. There are different types of containers:
- Vector: store content of the same data type. For example:

$>\quad b<-c(1,3.2,5.7)$

$>\mathrm{b}$

[1] $1.0 \quad 3.25 .7$

In this example, container b stores three pieces of data of the same type (numeric). Pay attention to the "[1]" before the content in the result: this is a numeric indicator of the position of the data inside the vector. Now try this:

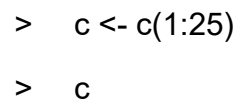

$\begin{array}{lrrrrrrrrrr}{[1]} & 1 & 2 & 3 & 4 & 5 & 6 & 7 & 8 & 9 & 10 \\ {[11]} & 11 & 12 & 13 & 14 & 15 & 16 & 17 & 18 & 19 & 20 \\ {[21]} & 21 & 22 & 23 & 24 & 25 & & & & & \end{array}$

First of all, the colon sign gives a range of the numbers between the two numbers that flank the sign. Secondly, you can now appreciate better that the numbers in the square brackets provide the position of the data in the vector.

By the way, the sign "<-," yeah, exactly, a "smaller" sign followed by a hyphen to make it look like a left-pointed arrow, is the sign that $R$ recognizes as putting the stuff on the right side to the left side, which is usually a container. Compare this syntax to a linear equation: $y=2+$ $3 x$, where $x=1$. In $R$, you will code as:

$>\quad x<-3$

$>\mathrm{y}<-2+3 * \mathrm{x}$

\# this step stores the result

in $y$, a container

$>\quad$ y \# this step calls the container $y$

[1] 11

- List: I have mixed feelings about lists. There are pros and cons. For the pros, lists are easy to use. You treat it like a plastic bag, into which you throw everything. Imagine that you just went to the grocery store. You bought a box of eggs, two bottles of milk, and a stack of cheese. You put them all into a giant bag. Whereas the box, the bottle, and the plastic wrap for the stack of cheese are all vectors (because inside these containers you have content of the same type), the bag is a list, which allows you to store all kinds of data, even if they are in different types. Now try this:

$>\quad$ my_list <- list(2, "neonatology", c(3.4, 5:8))

$>$ my_list

[[1]]

[1] 2

[[2]] 
[1] "neonatology"

[[3]]

[1] $\begin{array}{lllll}3.4 & 5.0 & 6.0 & 7.0 & 8.0\end{array}$

$>$ class(my_list)

[1] “list”

Now you get it, right? You have a container called my_list, which stores three objects: the first one is numeric data 2 , the second one is a character data "neonatology," the third is a vector with five numbers. When you check the container type with the function class(), you get a return saying that my_list is indeed a list. I use class() to check the type of container I am working with.

The cons for a list container type, as you probably noticed, is that the content in the list is segregated into various compartments. The numbers inside double square brackets are the relative position of the content in each compartment. How do we call the content inside each compartment and copy the content into another container?

$>$ new_list <- my_list[3]

the 3rd compartment inside new_list

\# place

$>$ new_list

[[3]]

[1] $3.4 \quad 5.0 \quad 6.0 \quad 7.0 \quad 8.0$

$>$ class(new_list)

list is still a list

\# new_

[1] "list"

$>$ another_new_list <- my_list[[3]]

the content of the 3rd compartment

\# inside another_new_list

[1] $\begin{array}{lllll}3.4 & 5.0 & 6.0 & 7.0 & 8.0\end{array}$

$>$ class(another_new_list)

er_new_list is a numeric vector

\#anoth-

[1] "numeric"

I hope you get the idea from this example. You use single square brackets to move the entire compartment into a new container; the new container is still a list. Or, you use double square brackets to move the content inside the compartment into a new container; the new container, which is a vector, will assume the type that $\mathrm{R}$ will try to coerce all the content into.

Now try this:

$>$ everything <- unlist(my_list)

$>$ everything

"5" "6" "2" "neonatology" "3.4"

[6] "7"

"8"

In this example, you use the function unlist() to break apart the compartments and put everything in a giant container named everything. $R$ will coerce everything into one data type, as the container everything is a vector (if you do not specify a container type, the default in $R$ is a vector type).

A final word on the list is that, just like vectors and lists have names, the compartments inside the list can be named and called upon by their names. Try this: list(HR $=\mathrm{c}(120,145,140), \mathrm{RR}=\mathrm{c}(24,35,34), \mathrm{FiO} 2=\mathrm{c}(0.21$, $0.22,0.33))$. We will talk more about it later.

- Dataframe: This is basically a two-dimensional table. To create a dataframe, do the following:

$>\mathrm{df}<$ - data.frame $(\mathrm{HR}=\mathrm{c}(120,145,140), \mathrm{RR}=\mathrm{c}(24$, $35,34), \mathrm{FiO} 2=\mathrm{c}(0.21,0.22,0.33))$

$>d f$

\begin{tabular}{llll} 
& HR & \multicolumn{2}{l}{ RRFiO2 } \\
1 & 120 & 24 & 0.21 \\
2 & 145 & 35 & 0.22 \\
3 & 140 & 34 & 0.33
\end{tabular}

A data frame has row numbers (they are actually stored as the character type), which you can change to row names by using the rownames() function. Each column may have a column name, which can be changed using the colnames() function.

To call content stored in a data frame, memorize this rule: container[row, column].

- To call the second row: $\mathrm{df}[2$,

- To call the second column: df[,2] or df[, "RR"]

- To call row 2 column 3: df[2,3]

A dataframe can store any type of data, but data in each column needs to be of the same data type. Think of this as having your research subjects in rows and the variables in columns:

\begin{tabular}{|l|l|l|l|}
\hline & HR & RR & FiO2 \\
\hline Subject 1 & & & \\
\hline Subject 2 & & & \\
\hline Subject 3 & & & \\
\hline
\end{tabular}

\section{Summary:}

In this post, we introduced common data types and data containers. These are the foundations of data collection and organization. 
Try to create different types of containers on your $\mathrm{R}$ console and compare them to your data on the Excel spreadsheets.

\section{"In this post, we introduced common data types and data containers. These are the foundations of data collection and organization. Try to create different types of containers on your $R$ console and compare them to your data on the Excel spreadsheets."}

\section{References:}

1. Hoyt RE, Hersh WR. Health Informatics: Practical Guide. Lulu Com; 2018. https://play.google.com/store/books/ details?id $=$ L1FewAEACAAJ

2. Wickham H, Grolemund G. Welcome. Accessed January 7, 2021. https://r4ds.had.co.nz/

Disclosure: The author identifies no conflict of interest

NT


\section{Neonatology Today's Digital Presence}

Neonatology Today's now has a digital presence. The site is operational now and defines the future look of our digital web presence. By clicking on this https://www. neonatologytoday.org/ web/., researchers can download individual manuscripts both in digital format and as part of the original PDF (print journal). While the PDF version of Neonatology Today will continue in its present form, we envision that the entire website will be migrated to this format in the next several months. We encourage you to take a look, "kick the wheels," and let us know where we still need to improve.. We are working towards making the website more functional for subscribers, reviewers, authors and anyone else. Although we have not yet applied for inclusion in the National Library of Medicine Database (Pub-Med), this new format meets several of the important metrics for this ultimate goal. As of December, 2020, NT has its own account with CrossRef and will assign DOI to all published material.

As we indicated last month, we look forward to a number of new features as well.

1. An online submission portal: Submitting a manuscript online will be easier than before. Rather than submitting by email, we will have a devoted online submission portal that will have the ability to handle any size manuscript and any number of graphics and other support files. We will have an online tracking system that will make it easier to track manuscripts in terms of where they are in the review process.

2. Reviewers will be able to review the manuscript online. This portal will shorten the time from receipt of review to getting feedback to the submitting authors.

3. An archive search will be available for journals older than 2012.

4. A new section called news and views will enable the submission of commentary on publications from other journals or news sources. We anticipate that this will be available as soon as the site completes the beta phase

5. Sponsors will be able to sign up directly on the website and submit content for both the digital and PDF issues of Neonatology Today.

Neonatology Today will continue to promote our Academic True Open Model (ATOM), never a charge to publish and never a charge to subscribe.

If there are any questions about the new website, please email Dr. Chou directly at:

fu-sheng.chou@neonatologytoday.net

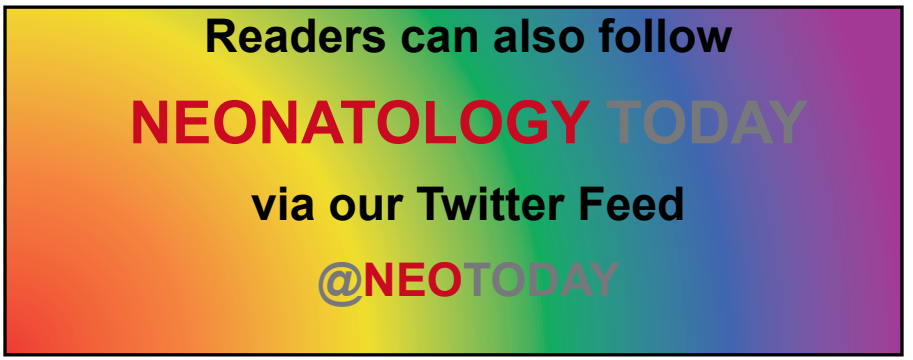

